\title{
Seeding Efficiency of Primitive Human Hematopoietic Cells in Nonobese Diabetic/Severe Combined Immune Deficiency Mice: Implications for Stem Cell Frequency Assessment
}

\author{
By Paula B. van Hennik, Alexandra E. de Koning, and Rob E. Ploemacher
}

\begin{abstract}
Nonobese diabetic/severe combined immune deficiency (NOD/SCID) mouse repopulating cells (SRC) have been proposed to represent a more primitive human stem cell subset than the cobblestone area-forming cell (CAFC) week (wk) 6 or the long-term culture-initiating cell (LTC-IC) wk 5 on the basis of their difference in frequency, phenotype, transfectibility, and multilineage outgrowth potential in immunodeficient recipients. We have assessed the percentage of various progenitor cell populations (colony-forming cell [CFC] and CAFC subsets) contained in unsorted NOD/SCID BM nucleated cells (nc), human umbilical cord blood (UCB) nc, bone marrow (BM) nc, peripheral blood stem cells (PBSC), and $\mathrm{CD}^{+}{ }^{+}$selected UCB nc, seeding in the BM and spleen of NOD/SCID mice within 24 hours after transplantation. The seeding efficiency of NOD/SCID BM CAFC wk 5 was median (range) in the spleen $2.9 \%(0.7 \%$ to $4.0 \%)$ and in the total BM
\end{abstract}

O VER THE PAST decade various in vitro and in vivo surrogate assays have been developed to estimate human stem cell frequencies. The cobblestone area-forming cell $(\mathrm{CAFC})^{1,2}$ and long-term culture-initiating cell (LTC-IC) ${ }^{3}$ assays have been proposed to determine and enumerate primitive progenitor cells with long-term repopulating ability in vitro in both the murine and human hematopoietic system. A quantitative in vivo assay for human hematopoietic stem cells has been established by the recent development of the nonobese diabetic/ severe combined immune deficiency (NOD/SCID) mouse model. ${ }^{4}$ In this model, human (stem) cells are infused into the tail vein of a sublethally irradiated NOD/SCID mouse, with optional intraperitoneal administration of cytokines to facilitate outgrowth of the stem cells that homed to the bone marrow. Thirty-five days after transplantation, the bone marrow (BM) cells of the NOD/SCID mice are collected and the percentage of human chimerism is determined using either flow cytometric analysis of the pan-leukocyte marker CD45 or Southern blot analysis for the human chromosome 17-specific $\alpha$-satellite probe. ${ }^{4}$ Stage- and/or lineage-specific antibodies are used to determine multilineage outgrowth. On the assumption that every infused SRC will generate detectable human engraftment, limiting dilution techniques have been used to establish the frequency of the SRC in umbilical cord blood (UCB), BM, and peripheral blood stem cells (PBSC), ie, UCB 1:930,000 nucleated cells (nc); BM 1:3,000,000 nc and PBSC 1:6,000,000 nc. ${ }^{5}$ Conneally et $\mathrm{al}^{6}$ obtained similar results in enumerating the frequency of the competitive repopulating units (CRU) in UCB. However, from syngeneic murine studies, it is known that only $18 \%$ to $20 \%$ of the infused stem cells home to the total BM and $8 \%$ to $10 \%$ lodge in the spleen. ${ }^{7}$ Additionally, it is still unknown whether all stem cells that reach the BM actually contribute to hematopoietic reconstitution of depleted marrow spaces. The cells that do not reach their niches in hematopoietic tissues are likely to be sequestered in organs with large capillary beds like the liver and lungs. ${ }^{8}$ The seeding efficiency of human stem cells in the NOD/SCID mouse could even be lower than in the
$8.7 \%(2.0 \%$ to $9.2 \%)$. For human unsorted UCB nc, BM nc, PBSC, and CD34+ UCB cells, the seeding efficiency for CAFC wk 6 in the BM of NOD/SCID mice was $4.4 \%$ (3.5\% to $6.3 \%)$, $0.8 \%(0.3 \%$ to $1.7 \%), 5.3 \%$ ( $1.4 \%$ to $13.6 \%)$, and $4.4 \%(3.5 \%$ to $6.3 \%)$, respectively. Using flow cytometry, the percentage CD34+ UCB cells retrieved from the BM of sublethally or supralethally irradiated NOD/SCID mice was 2.3 (1.4 to 2.8) and 2.5 (1.6 to 2.7), respectively. Because we did not observe any significant differences in the seeding efficiencies of the various stem cell subsets, it may be assumed that the SRC seeding efficiency in NOD/SCID mice is similarly low. Our data indicate that the seeding efficiency of a graft can be of great influence when assessing stem cell frequencies in in vivo repopulation assays.

(C) 1999 by The American Society of Hematology.

syngeneic murine situation. This implicates that the seeding efficiency of a graft can be of great influence when assessing stem cell frequencies in repopulation assays. To estimate the seeding efficiency of human stem cells in the NOD/SCID mouse, we have assessed the percentages of CAFC subsets and colony-forming cell (CFC) that home to the BM and spleen of the NOD/SCID mouse within the first 24 hours postinfusion. Additionally, we have studied the seeding efficiency of CD34 ${ }^{+}$ selected UCB cells using in vitro culture assays, as well as flow cytometric analysis.

\section{MATERIALS AND METHODS}

Human cells. In the described experiments, cells from different human grafts were used. The UCB samples were obtained from umbilical cords from full-term, healthy newborns. The cord blood cells were 1:1 diluted with Hanks' Balanced Salt Solution (HBSS; GIBCO, Breda, The Netherlands) and processed using either a Ficoll-gradient $\left(1.077 \mathrm{~g} / \mathrm{cm}^{2}\right.$; Nycomed, Oslo, Norway) or a $3 \%$ gelatine solution (Sigma, St Louis, MO). After harvesting, the mononuclear cell fraction or the erythrocyte-depleted fraction, respectively, the cells were washed twice in HBSS. The BM nc were obtained by posterior iliac crest puncture from 5 hematological healthy adults. The mononuclear cell fraction was isolated from the BM cells using a Ficoll-gradient as

From the Institute of Hematology, Erasmus University Rotterdam, Rotterdam, The Netherlands.

Submitted February 16, 1999; accepted June 28, 1999.

Supported by Grant No. 95-1020 from the Dutch Cancer Society.

Address reprint requests to Rob E. Ploemacher, PhD, Institute of Hematology, Room Ee 1391, Erasmus University Rotterdam, Dr. Molenwaterplein 50, PO Box 1738, 3000 DR Rotterdam, The Netherlands; e-mail:ploemacher@hema.fgg.eur.nl.

The publication costs of this article were defrayed in part by page charge payment. This article must therefore be hereby marked "advertisement" in accordance with 18 U.S.C. section 1734 solely to indicate this fact.

(C) 1999 by The American Society of Hematology.

0006-4971/99/9409-0020\$3.00/0 
described above. The PBSC were obtained from 5 patients with non-Hodgkin's lymphoma (NHL) and 1 patient with multiple myeloma (MM). The mononucleated cell fraction was isolated using a Ficollgradient. These patients were in complete remission. The patients, the allogeneic donors, and the mothers of the newborns gave their informed consent. The UCB nc, BM nc, and PBSC were cryopreserved until use in 10\% dimethyl sulfoxide (DMSO; BDH, Poole, UK) and 20\% heat-inactivated fetal calf serum (FCS; Summit Biotechnology, Fort Collins, CO). The unsorted human stem cells were used for transplantation into 9 Gy irradiated NOD/SCID mice, CAFC, and CFC cultures.

NOD/SCID mouse BM cells for transplantation. Male and female specific pathogen-free NOD/LtSz-scid/scid (NOD/SCID) mice, 3 to 5 weeks of age, were obtained from the Department of Immunology at the Erasmus University Rotterdam. The mice were included in experiments at the age of 6 to 9 weeks. To obtain NOD/SCID BM cells for transplantation, in total $15 \mathrm{NOD} / \mathrm{SCID}$ mice were killed using $\mathrm{CO}_{2}$ asphyxiation and both femora and tibia were removed. The NOD/SCID BM cells were harvested using crunching of the bones in HBSS with 5\% FCS. The cells were collected and sieved through a $100-\mu \mathrm{m}$ sieve. After centrifugation at 1,800 rpm for 8 minutes, the cells were resuspended in phosphate-buffered saline (PBS; GIBCO). The NOD/SCID BM cells were used for transplantation into 9 Gy irradiated NOD/SCID mice, CAFC, and CFC cultures.

Isolation of $\mathrm{CD} 34^{+} \mathrm{UCB}$ cells. The mononucleated cell fraction of UCB samples was thawed and using the Macs-selection system (Miltenyi Biotec $\mathrm{GmbH}$, Bergisch Gladbach, Germany), $\mathrm{CD}^{+} 4^{+}$cells were isolated according to the manufacturer's instructions. The purity ranged from $72 \%$ to $99 \%$. The $\mathrm{CD} 34^{+} \mathrm{UCB}$ cells were used for transplantation into 3.5 or 9 Gy irradiated NOD/SCID mice and flow cytometric analysis.

$N O D / S C I D$ repopulating cell (SRC) frequency analysis in UCB. To estimate the frequency of the SRC in UCB, 5 to 21 samples were pooled and intravenously (IV) transplanted into sublethally irradiated NOD/ SCID mice using 9 cell concentrations ranging from 1,660 to 225,000 $\mathrm{CD} 34^{+} \mathrm{UCB}$ cells per mouse. Per cell dose, the median number of mice used was 6 (2 to 34). After 35 days, the femora were isolated and BM cells harvested. The percentage of multilineage engraftment was determined using immunophenotypic analysis for each individual mouse. Mice containing $1 \%$ or more $\mathrm{CD} 45^{+}$cells were considered positive for human engraftment. During the engraftment period, ie, 35 days posttransplant, the mice were not supplemented with cytokines. The frequency of the SRC in UCB in our laboratory was determined by using Poisson statistics. Part of the repopulation data used has been published by Verstegen et al. ${ }^{9}$

Assessment of the seeding efficiency of the transplanted cells. For determining the seeding efficiency in NOD/SCID mice of all 5 grafts studied, we determined the number of CAFC subsets and CFC per graft. Simultaneously, we irradiated 2 recipient NOD/SCID mice per sample with 9 Gy by a ${ }^{137} \mathrm{Cs}$ source (Gammacell; Atomic Energy of Canada, Ottawa, Canada). This dose was chosen to maximally reduce the recipient hematopoietic activity, allowing the detection of low numbers of human seeded stem cells. Two to 5 hours after irradiation, the mice were transplanted. In the experiments assessing the seeding of CD34 ${ }^{+}$ UCB cells as determined by flow cytometry, the mice were either 3.5 or 9 Gy irradiated. The number of transplanted cells per mouse varied in case of unsorted UCB between 63 and $111 \times 10^{6} \mathrm{nc}$, for BM between 30 and $48 \times 10^{6} \mathrm{nc}$, for PBSC between 30 and $94 \times 10^{6} \mathrm{nc}$, and for NOD/SCID BM between 46 and $63 \times 10^{6} \mathrm{nc}$. The median percentage of $\mathrm{CD}^{+} 4^{+}$cells in the unsorted UCB graft was 1.7 , in BM 5.3, and in PBSC 1.5. For the $\mathrm{CD}_{3} 4^{+}$grafts, the cell numbers varied between 375,000 and 800,000 pure $\mathrm{CD} 34^{+}$nc per mouse. In case of transplanting unsorted UCB, BM, PBSC, NOD/SCID BM, and CD34+ selected UCB nc 3, 5, 6, 4, and 7 individual samples have been used, respectively. At 22 to 24 hours after transplantation, ${ }^{10,11}$ the mice were killed and femora and spleen were isolated. The BM cells were harvested by crunching the bones as described above. The spleen was carefully cut in parts and sieved through a $100-\mu \mathrm{m}$ mesh filter. The cells were then washed and resuspended in CAFC medium or PBS containing $0.5 \%$ bovine serum albumin (BSA) and $2 \%$ normal human serum (NHS; obtained from healthy volunteers) in case of preparing the cells for immunophenotyping. In the CAFC assay, $1 / 10$ femur or $1 / 50$ spleen were plated in the first dilution. For the CFC assay, 1/10 and 1/100 femur and $1 / 50$ and $1 / 500$ spleen were plated. The irradiation control was negative in 6 of 6 experiments. For determining the seeding efficiency to the total BM, we assumed that 1 femur contains approximately $6 \%$ of the total BM cellularity. ${ }^{12}$ The BM and spleen seeding efficiencies were calculated on the basis of the number of infused and retrieved CAFC and CFC. When CD34 ${ }^{+}$UCB cells were infused and the phenotypic seeding efficiency was determined, the number of retrieved $\mathrm{CD} 34^{+}$UCB cells (as determined using Flow Count Fluorospheres; Coulter Immunotech, Miami, FL) was divided by the number of CD34 $4^{+} \mathrm{UCB}$ cells infused corrected for the purity of the population. Seeding efficiency is expressed as percentage.

Immunofluorescence analysis. Cells were stained with anti-CD34fluorescein isothiocyanate (FITC) or phycoerythrin (PE), anti-CD45FITC (Coulter Immunotech, Mijdrecht, The Netherlands) or antiCD38-PE (Becton Dickinson, San Jose, CA) or FITC (Coulter Immunotech) by incubating $10^{5}$ to $10^{6} \mathrm{nc}$ for 30 minutes on ice. The incubations were performed in PBS containing 0.5\% BSA (Sigma) and $2 \%$ NHS. After incubation, the cells were washed once in PBS and $0.5 \%$ BSA and resuspended in $0.3 \mathrm{~mL}$ PBS. Analysis was performed using a Facscan (Becton Dickinson). A total of 10 to 25,000 events was acquired per sample. In case of using flow cytometry for determining the seeding efficiency of $\mathrm{CD} 34^{+}$selected UCB cells in the BM of the NOD/SCID mouse, a minimum of 750,000 events was acquired. Seven-aminoactinomycin D (7-AAD; Molecular Probes, Eugene, OR) was used to exclude the dead cells.

Hematopoietic growth factors. Purified recombinant human granulocyte-macrophage colony-stimulating factor (GM-CSF) and murine stem cell factor (SCF) were kindly provided by Genetics Institute (Cambridge, MA). Human granulocyte colony-stimulating factor (GCSF) and human interleukin-3 (IL-3) were gifts from Amgen (Thousand Oaks, CA) and Gist Brocades (Delft, The Netherlands), respectively.

Human CFC assay. Quantification of the number of colonyforming units-granulocyte macrophage (CFU-GM) and burst-forming units-erythroid (BFU-E) was performed using a semisolid (1.2\% methylcellulose; Methocel, Stade, Germany) culture medium (Iscove's modified Dulbecco's medium [IMDM]; GIBCO) at $37^{\circ} \mathrm{C}$ and $5 \% \mathrm{CO}_{2}$. The cultures contained $30 \%$ FCS supplemented with penicillin (100 $\mathrm{U} / \mathrm{mL}$; GIBCO), streptomycin $(100 \mu \mathrm{g} / \mathrm{mL}$; GIBCO), $\beta$-mercaptoethanol ( $\beta \mathrm{me} ; 5 \times 10^{-5} \mathrm{~mol} / \mathrm{L}$; Merck, Darmstadt, Germany), erythropoietin (1 U/mL; Boehringer, Mannheim, Germany), IL-3 (15 ng/mL), G-CSF (50 ng/mL), GM-CSF (5 ng/mL), and murine SCF (100 ng/mL) all at final concentrations. CFU-GM and BFU-E consisting of more than 50 cells were counted on day 14 of culture in the same dish.

Murine CFC assay. The number of CFC present in the murine BM and spleen cells harvested was determined by plating the cells in semisolid cultures consisting of $1.2 \%$ (wt/vol) methylcellulose (Methocel) in IMDM. The medium was supplemented with $10 \%$ (vol/vol) pokeweed mitogen-stimulated mouse spleen-conditioned medium and $20 \%$ horse serum (HS; GIBCO). The cultures were kept at $37^{\circ} \mathrm{C}$ and $10 \% \mathrm{CO}_{2}$. Colonies consisting of 50 cells and more were counted at day 7 and day 14 of culture.

Stromal feeders. The Flask Bone Marrow Dexter (FBMD-1) murine stromal cell line was used as described earlier. ${ }^{2}$ In short, stromal feeders were prepared by seeding $10^{3}$ FBMD- 1 cells per well into flat-bottom 96-well plates (Falcon, Lincoln Park, NJ) from log-phase cultures. Culture plastics destined for establishment of FBMD-1 stromal feeders were incubated overnight at $4^{\circ} \mathrm{C}$ with $0.3 \%$ gelatin in 
demineralized water to improve adherence of the stromal layer. The FBMD-1 cells were cultured in FBMD-1 medium consisting of IMDM with glutamax-1 (GIBCO) supplemented with penicillin (100 U/mL), streptomycin $(100 \mu \mathrm{g} / \mathrm{mL}), \beta \mathrm{me}\left(10^{-4} \mathrm{~mol} / \mathrm{L}\right), 10 \% \mathrm{FCS}, 5 \%$ HS (Integro, Zaandam, The Netherlands), and hydrocortisone 21 -hemisuccinate $\left(10^{-5} \mathrm{~mol} / \mathrm{L}\right.$; Sigma). After 7 to 10 days of culture at $33^{\circ} \mathrm{C}$ and $10 \% \mathrm{CO}_{2}$, the stromal layers had reached confluence and were used for the CAFC assay.

$C A F C$ assay. Confluent stromal layers of FBMD-1 cells in flatbottom 96-well plates were overlaid with NOD/SCID BM nc, UCB nc (unsorted or $\mathrm{CD} 34^{+}$selected), BM nc, or PBSC in a limiting dilution setup. For the primary CAFC assays of the NOD/SCID BM nc, UCB nc, $\mathrm{BM} \mathrm{nc}$, and PBSC, the input values ranged from 27,000 to 50,000 nc per well and 250 cells per well in case $\mathrm{CD} 34^{+} \mathrm{UCB}$ cells. The portion of the harvested BM and spleen of the transplanted NOD/SCID mice used in the first dilution of the CAFC assay is indicated above. Twelve dilutions, 2-fold apart were used for each sample with 15 replicate wells per dilution. The cells were cultured at $33^{\circ} \mathrm{C}$ and $10 \% \mathrm{CO}_{2}$ for 5 weeks in case of mouse cells and 6 weeks for the human CAFC with weekly half-medium changes. For the murine CAFC assay, FBMD-1 medium was used and in case of performing the human CAFC assay, the medium consisted of FBMD-1 medium supplemented with IL-3 and G-CSF at final concentrations of $10 \mathrm{ng} / \mathrm{mL}$ and $20 \mathrm{ng} / \mathrm{mL}$, respectively. The percentage of wells with at least 1 phase-dark hematopoietic clone of at least 5 cells (ie, cobblestone area) beneath the stromal layer was determined weekly for the mouse CAFC and every 2 weeks for the human CAFC. Frequencies of the CAFC subsets were calculated using Poisson statistics as described previously. ${ }^{1}$

Data analysis. Microsoft Excel 97 (Microsoft, Redmond, WA) and SPSS for Windows Release 7.5.2. (SPSS Inc, Chicago, IL) were used for data analysis. Data are expressed as median (range). Statistical comparisons were performed according to Mann Whitney U-test. The 2-sided $P$ value was determined testing the null hypothesis that the 2 population medians are equal. $P$ values $<.05$ were considered significant.

\section{RESULTS}

$S R C$ frequency in $U C B$. The SRC frequency as determined by limiting dilution analysis in our laboratory is approximately 1 per $6.6 \times 10^{6}$ unsorted UCB nc. The threshold for human engraftment used to distinguish positive from negative mice is $1 \% \mathrm{CD} 5^{+} \mathrm{nc}$ in the BM of the NOD/SCID mouse as assessed by flow cytometry.

Low seeding efficiency of NOD/SCID BM cells in NOD/SCID mouse BM and spleen. To determine the seeding efficiency of syngeneic hematopoietic cells in the NOD/SCID mouse strain, NOD/SCID BM nc were isolated from 6- to 9-week-old NOD/SCID mice and processed according to the seeding efficiency protocol as described in Materials and Methods. The seeding efficiency to the total BM of the NOD/SCID mouse tended to decrease with increasing immaturity of the progenitor cell subset studied (Fig 1). The median (range) seeding efficiency to the total BM for CFC day 7 (d 7) and d 14 was 19.5 (17.6 to 24.1 ) and 22.8 (13.1 to 28.4), respectively. In contrast, the seeding efficiency of CAFC wk 5 was 8.7 (2.0 to 9.2). For the seeding efficiency of the progenitor subsets to the murine spleen, we did not observe significant differences. The seeding efficiency of d $7 \mathrm{CFC}$ and d $14 \mathrm{CFC}$ to the spleen was 1.4 (0.5 to 2.4 ) and 1.2 (0.9 to 2.5), respectively. The percentage of CAFC wk 5 lodging to the spleen was 2.9 (0.7 to 4.0 ).

Seeding efficiency of unsorted human progenitors in total BM and spleen of NOD/SCID mice. All of the progenitor subsets analyzed from unsorted UCB nc showed a lower seeding efficiency to the BM and the spleen than was observed in the NOD/SCID syngeneic setting (Fig 2). The seeding efficiency of d 14 CFC and CAFC wk 6 to the total BM of the NOD/SCID mouse was in case of unsorted UCB nc 3.8 (3.8 to 5.5) and 4.4 (3.5 to 6.3), in BM nc 0.9 (0.1 to 1.4) and 0.8 (0.3 to 1.7), and in PBSC 2.1 (0.1 to 3.9) and 5.3 (1.4 to 13.6), respectively. The $d$ $14 \mathrm{CFC}$ and CAFC wk 6 seeding efficiency to the spleen was in case of unsorted UCB nc 0.7 (0.6 to 1.0) and 0.5 (0.4 to 0.8 ), for BM nc 0.3 (0.03 to 0.9$)$ and 0.2 (0.1 to 0.2), and for PBSC 0.6 (0.1 to 1.2 ) and 0.5 (0.1 to 1.8 ). Thus, of all infused progenitors derived from, for example, unsorted UCB nc, only around 4 of every $100 \mathrm{CAFC}$ infused will home to the total BM and only 5 in every 1,000 will lodge to the spleen of the NOD/SCID mouse. The seeding efficiency of the various hematopoietic progenitor cell types from PBSC was comparable to the seeding efficiency of UCB nc, both to total BM and spleen (Fig 2). However, PBSC grafts showed larger variability in seeding to $\mathrm{BM}$ and spleen as compared with UCB grafts. As with UCB cells, no significant differences could be observed among the subsets assayed.

Also the seeding efficiency of human BM progenitors to the murine BM and the spleen showed no significant differences in seeding efficiency among the various stem cell subsets studied.

Seeding efficiency of $C F C$ and CAFC subsets in $C D 34^{+}$ selected UCB cells. As it may be argued that the seeding efficiency may change in relation to the cell number infused, we have compared the seeding efficiency of CFC and CAFC subsets from $\mathrm{CD}_{3} 4^{+}$selected and unsorted UCB cells (Fig 3). No significant difference could be observed. As observed with the unsorted grafts, the different progenitor subsets contained in the $\mathrm{CD} 34^{+}$grafts home in comparable percentages to the $\mathrm{BM}$ of the NOD/SCID mouse.
Fig 1. Seeding efficiency of unsorted NOD/SCID BM cells in total bone marrow $(\square)$ and spleen $(O)$ of NOD/SCID mice. The data represent the median and range of 4 separate experiments.

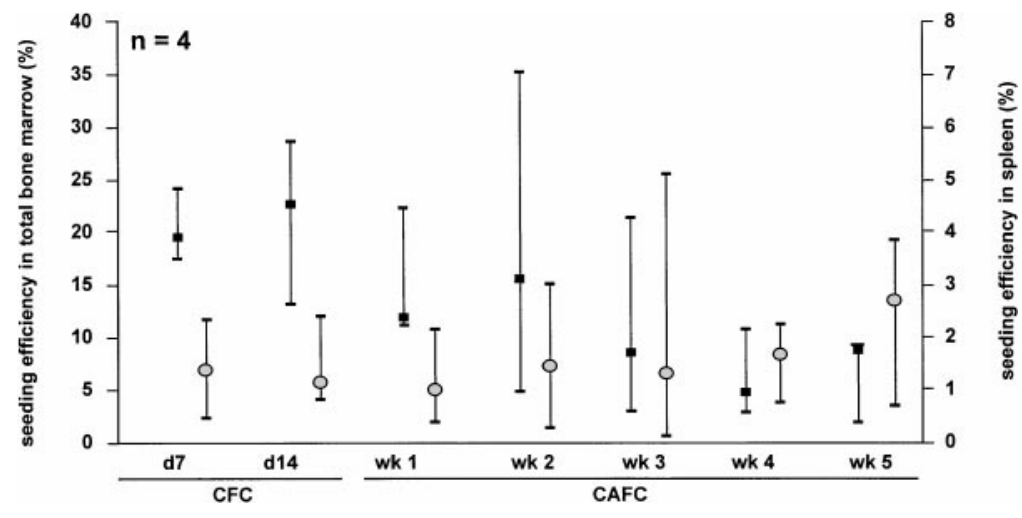




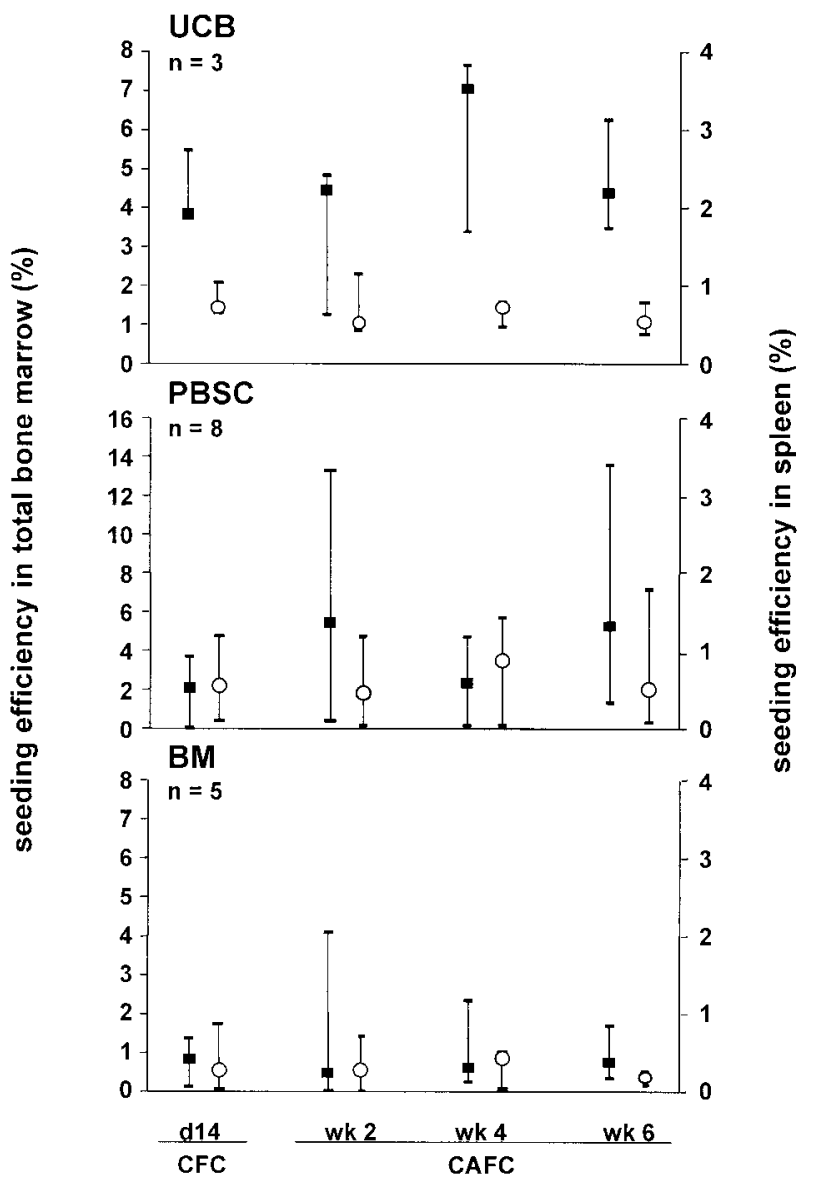

Fig 2. Seeding efficiency of human unsorted UCB nc, PBSC, and BM nc in total NOD/SCID BM $(\square)$ and spleen $(O)$. The data are depicted as median and range. $n$, number of separate experiments.

The effect of 3.5 Gy versus 9 Gy irradiation conditioning on the seeding efficiency of $\mathrm{CD} 34^{+} \mathrm{UCB}$ cells as determined using flow cytometric analysis. Determination of the repopulating potential of human cells in the NOD/SCID mouse model commonly includes conditioning using a sublethal total body irradiation of $3.5 \mathrm{~Gy}$. In our experiments to determine the seeding efficiency of human grafts in the NOD/SCID mouse, we irradiated the mice with a supralethal irradiation of 9 Gy to fully eradicate any measurable constitution by murine progenitors. This dose of irradiation would then assure detection of human progenitor cells exclusively, as we are not able to distinguish murine and human progenitors using the CFC and CAFC assay as used here. To assess whether the level of irradiation affected the seeding efficiency of human progenitors in the NOD/SCID mice, we compared the seeding efficiency of $\mathrm{CD} 34^{+}$selected UCB cells as assessed by flow cytometry in 3.5 Gy and 9 Gy irradiated NOD/SCID mice. Figure 4 shows the flow cytometric analysis used to determine the number of $\mathrm{CD} 34^{+} \mathrm{UCB}$ cells retrieved from the NOD/SCID mouse BM 24 hours after transplantation. In dot plot $\mathrm{A}$, living, ie, 7-AAD negative, CD34-PE positive cells are gated and shown in dot plot B. The CD45 ${ }^{\text {dim }}$ cells possessing large forward light scatter properties were gated to prevent false positive events due to the high autofluorescence of murine BM cells and shown in a CD45FITC versus CD34-PE dot plot (C). Dot plot $\mathrm{C}$ shows a clear double positive population representing the $\mathrm{CD} 34^{+} \mathrm{UCB}$ cells that had homed to the NOD/SCID mouse BM.

Figure 5 shows that the median seeding efficiency of $\mathrm{CD} 34^{+}$ UCB cells in 3.5 Gy irradiated recipients $(2.3 \%)$ as compared with 9 Gy conditioned NOD/SCID (2.5\%) mice as measured by flow cytometry was not significantly different. Furthermore, the seeding efficiency of $\mathrm{CD}_{3}{ }^{+}$selected UCB cells as determined by the $\mathrm{CFC}$ and the CAFC assay is similar to that assessed by flow cytometric analysis except for the CAFC wk $6(P=.04)$. However, in $\mathrm{CD} 4^{+}$selected UCB cells, the CAFC wk 2 frequency relates to the CAFC wk 6 frequency as 4:1. Therefore, the seeding efficiency of $\mathrm{CD} 34^{+} \mathrm{UCB}$ cells as determined using flow cytometry will be in the range of the CAFC wk 2 seeding.

\section{DISCUSSION}

We have shown that the seeding efficiency of human progenitor cells in the BM and spleen of NOD/SCID mice is extremely low. As the different progenitor cell subsets had comparable low seeding rates, it is conceivable by extrapolation that only a few of all infused NOD/SCID repopulating cells will home to the marrow of these mice. Thus, the assumption that every SRC infused will indeed contribute to repopulation should be critically met. ${ }^{5}$ Rather, our data indicate that the published frequency estimates of human SRC in UCB, BM, and $\mathrm{PBSC}$ represent a dramatic underestimation. If these frequency

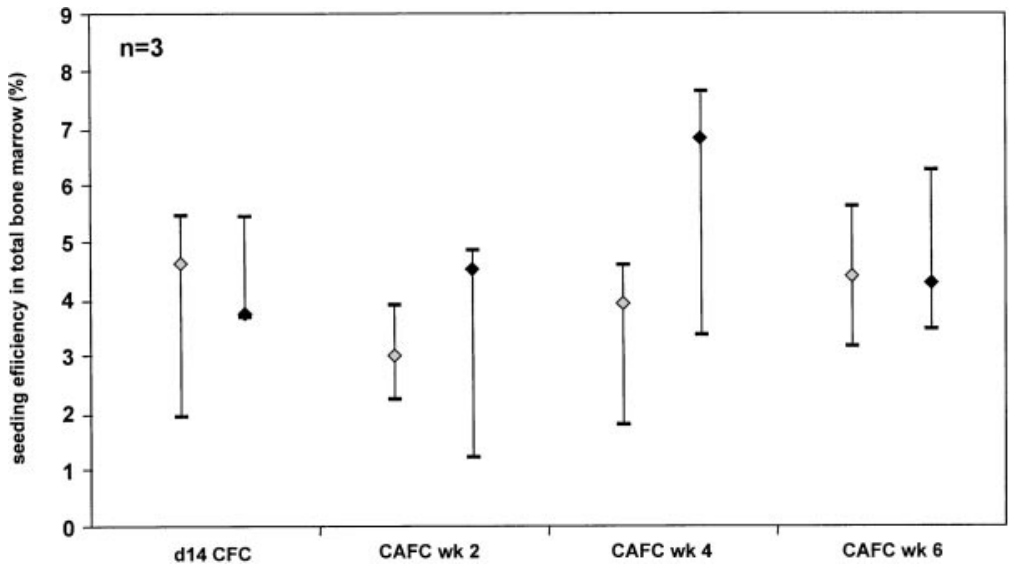

Fig 3. Comparison of the seeding efficiency of human $\mathrm{CD}^{+} 4^{+}$selected $(\diamond)$ and unsorted $(\diamond)$ UCB cells in total NOD/SCID BM. The data are depicted as median and range. Three separate experiments for either population were performed. 


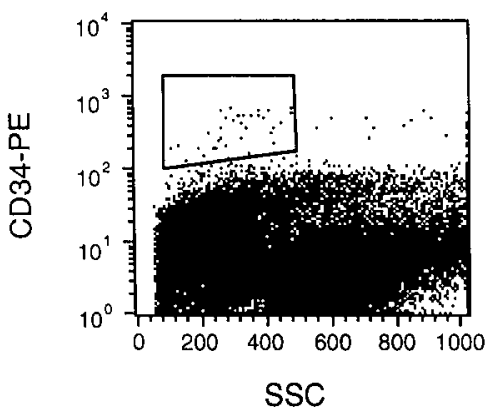

A.

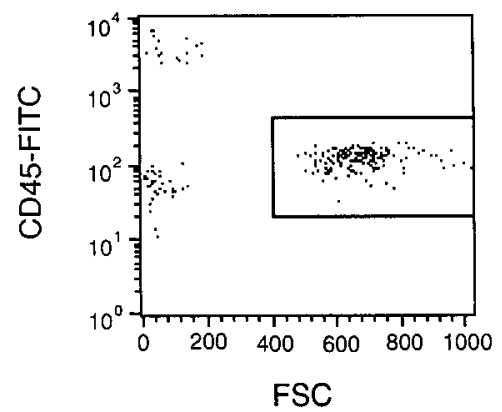

B.

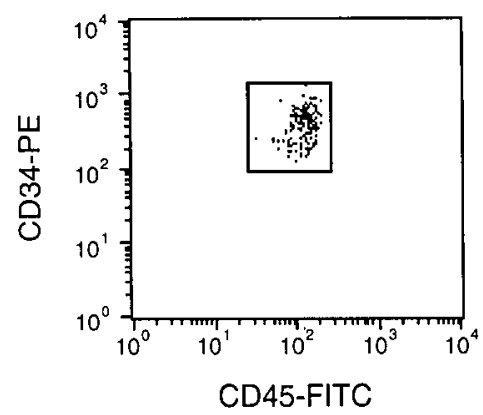

C.

Fig 4. Flow cytometric analysis used to determine the number of CD34+ UCB cells retrieved from the NOD/SCID mouse BM 24 hours after transplantation. Dead cells were excluded using 7-AAD. In dot plot A, living CD34-PE positive cells are gated and shown in dot plot B. CD45dim cells possessing large forward light scatter properties are gated and shown in a CD45-FITC versus CD34-PE dot plot (C). Dot plot C clearly shows that all of the gated cells in plot B belong to a clear double positive population representing the CD34+ UCB cells retrieved from the NOD/SCID mouse BM.

estimates were corrected by the respective seeding efficiencies as currently published, they would be 18 -fold to 125 -fold higher than presently accepted.

We have used $1 \% \mathrm{CD}^{2} 5^{+}$as a threshold for human engraftment in BM of the NOD/SCID mouse to distinguish positive from negative mice. This threshold is 10 -fold and 20 -fold higher than the threshold used by Conneally et $\mathrm{al}^{6}$ and Wang et al, ${ }^{5}$ respectively. The lower detection threshold used by Wang et al and Conneally et al may explain why these investigators arrived at a higher SRC frequency estimate.

The seeding efficiency of NOD/SCID BM nc to the BM and the spleen of a syngeneic recipient is lower than we have observed in other murine syngeneic transplantation settings. ${ }^{7}$ Particularly, the seeding efficiency to the spleen is strikingly low. The reason for this discrepancy is as yet unresolved; however, there are several possible explanations for this observation. (1) There could be intrinsic differences of the primitive stem cells between the 2 mouse strains influencing their homing behavior. (2) The data could indicate that the microenvironment in the NOD/SCID mouse may be less conducive for allowing homing of infused stem cells.

We observed similar seeding efficiencies to the NOD/SCID $\mathrm{BM}$ of CFC and CAFC subsets, irrespective of whether they were contained in $\mathrm{CD}_{3} 4^{+}$selected or unsorted UCB cells. These data suggest that CD34- UCB cells in the unsorted grafts did not affect the homing of the progenitor subsets contained in the $\mathrm{CD} 34^{+}$population.

By using flow cytometry, we were able to compare the seeding efficiency of $\mathrm{CD} 34^{+}$selected UCB cells in 3.5 Gy and 9 Gy irradiated NOD/SCID mice. Furthermore, the seeding efficiency of $\mathrm{CD} 34^{+}$selected UCB cells obtained with immunophenotypic analysis was not significantly different from the data obtained with the CFC and CAFC assays (except for CAFC wk 6). Although the data are highly suggestive, they do not exclude the possibility that $\mathrm{CD} 34^{+} \mathrm{UCB}$ cells show differences in cloning efficiency in the CFC and CAFC assay after seeding in $\mathrm{BM}$ of a 3.5-Gy irradiated mouse as compared with seeding in BM of a 9-Gy irradiated recipient.

Homing of stem and progenitor cells to the BM after IV transplantation has been defined as the cells' ability to seek marrow stroma selectively, to subsequently lodge within it, and initiate hematopoiesis. ${ }^{13}$ As investigated in the murine system, this process is believed to include 2 phases. The first phase consists of the recognition of the endothelial cells by the hematopoietic cells possibly through a lectin receptor with galactosyl specificity. ${ }^{14,15}$ The second phase is the interaction of galactosyl/mannosyl-specific homing receptor to the extracellular matrix of the $\mathrm{BM}^{16}$ and the interaction of the very late antigen-4 (VLA-4) receptor with vascular-cell-cell adhesion molecule (VCAM). ${ }^{17-19}$ Therefore, the process of homing seems
Fig 5. Seeding efficiency of human $\mathrm{CD}^{+} 4^{+} \mathrm{UCB}$ cells in total BM of NOD/SCID mice: comparison of flow cytometric analysis with CFC and CAFC data. The data are depicted as median and range of 3 separate experiments for the CFC and CAFC assays; 6 experiments for the 3.5-Gy irradiated NOD/SCID mice, and 7 experiments for the 9-Gy irradiated NOD/SCID mice. *, Significantly different from CAFC wk 6.

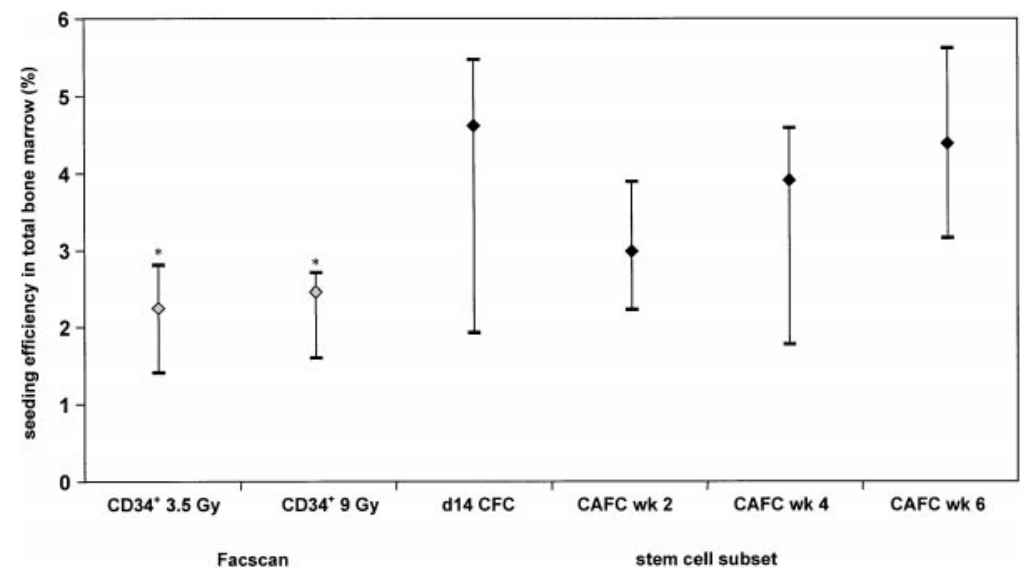


to be highly specific. In other organs (ie, liver, lung, and kidney), hematopoietic progenitor cells can be temporarily detected after their IV transplantation and disappear within 48 hours after transplantation. ${ }^{20}$ Their sequestration to the latter organs may be determined by other mechanisms, including binding to galactosyl receptors. ${ }^{21}$

Also the homing process to the spleen seems to be regulated differently than in the marrow. Recognition of galactosyl/ mannosyl residues ${ }^{16}$ or the fibronectin receptor (VLA-4) ${ }^{20}$ seems not to be involved. The special anatomical structure of the spleen is suggested to be one of the determinants of spleen cell homing.

In our experiments, we have injected human cells into a sublethally and supralethally irradiated NOD/SCID mouse. Several publications indicate that the molecules involved in the homing process are highly conserved in evolution. ${ }^{22-24}$ Therefore, it is likely that the transplanted human cells will home to the BM and spleen of the NOD/SCID mouse in a specific manner.

Our data indicate otherwise. As the total BM of a mouse may be estimated to weigh around $350 \mathrm{mg}$, while an NOD/SCID mouse 9 weeks old weighs around $18 \mathrm{~g}$, the total BM is around $2 \%$ of the total weight of the animal. The weight of the spleen is around $1 \%$ of the animal weight. If the homing of the human cells is random, then the seeding efficiency to the total BM will be $2 \%$ and to the spleen $1 \%$. However, when we take into account that tissues like fat, skin, and brain of the mouse are probably less accessible for progenitor cells, while additionally the capillary beds of the BM and the spleen may be rather extensive, we will expect a slightly higher seeding efficiency than these figures if seeding is a nonspecific process. In agreement with the calculated seeding percentages, the median seeding efficiencies of the stem cells in the human grafts studied to the total $\mathrm{BM}$ vary between $1 \%$ and $7 \%$ and to the spleen between $0.2 \%$ and $0.9 \%$. These data suggest that human progenitors do not home preferentially to the total BM and spleen of the NOD/SCID mouse, but that seeding of human hematopoietic progenitors may predominantly occur on the basis of organ-weight and capillary bed complexity.

The expression of adhesion molecules is different among $\mathrm{CD}_{3}{ }^{+}$cells derived from UCB and BM as compared with PBSC. PBSC shows lower expression of VLA-4, leukocyte function anigen-1 (LFA-1), intercellular adhesion molecule-1 (ICAM-1), and LFA-3 than BM. ${ }^{25,26}$ The adhesion molecule expression pattern in UCB nc shows similarities with BM nc, although UCB-derived $\mathrm{CD} 34^{+} / \mathrm{CD} 38^{-}$cells express higher levels of L-selectin (CD62L) and LFA-1 (CD11a) than their BM counterparts. ${ }^{27}$ This would mean that if the homing process of the human cells in the NOD/SCID mouse is specific, we might expect comparable seeding efficiencies of UCB and BM nc and a lower seeding of PBSC. In contrast, the seeding efficiencies of UCB nc and PBSC are comparable, while the seeding of BM nc in the BM of the NOD/SCID mouse is very low.

It has been published that expression of the molecules that recognize galactosyl and mannosyl residues changes during differentiation. ${ }^{28}$ This could cause differences in seeding efficiencies among progenitor and stem cell subsets. However, the fact that we did not observe this provides us with another strong indication that homing of the human cells in the BM and the spleen of the NOD/SCID mouse is not specific.

Our data suggest that the postulated low frequencies of human long-term repopulating stem cells may largely result from their low seeding efficiency in the BM of NOD/SCID mice. In addition, it should be realized that the proposed SRC frequency in the various human grafts is the resultant of (1) seeding efficiency, (2) repopulating ability of the transplanted cells, (3) exogenous factors facilitating engraftment, (4) detection methods, (5) detection thresholds, and (6) definition of a SRC. In light of this notion, the estimated human stem cell frequency could even be higher than the SRC frequency corrected for the seeding efficiency of that particular graft.

It has been recently suggested that the SRC may represent a different and more primitive stem cell subset than are assessed by the LTC-IC and CAFC assay on the basis of a variety of observations. ${ }^{5}$ Thus, SRC would be more difficult to transduce and occur at far lower frequencies than the LTC-IC or CAFC wk 6. Our recent data on comparable gene expression in CAFC wk 6 and SRC, ${ }^{29}$ together with our present data, would suggest that the SRC and CAFC wk 6 may differ far less than previously claimed and thus may represent overlapping stem cell populations.

\section{ACKNOWLEDGMENT}

The authors thank Dr A.Th. Alberda and the staff of the St Franciscus Hospital (Rotterdam, The Netherlands) for collection of cord blood samples used in this study. We also acknowledge Els van Bodegom for taking care of the NOD/SCID mice. Drs M.M.A. Verstegen and N. Kusadasi are gratefully acknowledged for sharing their data for SRC frequency analysis.

\section{REFERENCES}

1. Ploemacher RE, van der Sluijs JP, Voerman JS, Brons NH: An in vitro limiting-dilution assay of long-term repopulating hematopoietic stem cells in the mouse. Blood 74:2755, 1989

2. Breems DA, Blokland EA, Neben S, Ploemacher RE: Frequency analysis of human primitive haematopoietic stem cell subsets using a cobblestone area forming cell assay. Leukemia 8:1095, 1994

3. Sutherland HJ, Lansdorp PM, Henkelman DH, Eaves AC, Eaves CJ: Functional characterization of individual human hematopoietic stem cells cultured at limiting dilution on supportive marrow stromal layers. Proc Natl Acad Sci USA 87:3584, 1990

4. Lapidot T, Pflumio F, Doedens M, Murdoch B, Williams DE, Dick JE: Cytokine stimulation of multilineage hematopoiesis from immature human cells engrafted in SCID mice. Science 255:1137, 1992

5. Wang JC, Doedens M, Dick JE: Primitive human hematopoietic cells are enriched in cord blood compared with adult bone marrow or mobilized peripheral blood as measured by the quantitative in vivo SCID-repopulating cell assay. Blood 89:3919, 1997

6. Conneally E, Cashman J, Petzer A, Eaves C: Expansion in vitro of transplantable human cord blood stem cells demonstrated using a quantitative assay of their lympho-myeloid repopulating activity in nonobese diabetic-scid/scid mice. Proc Natl Acad Sci USA 94:9836, 1997

7. Van der Loo JC, Ploemacher RE: Marrow- and spleen-seeding efficiencies of all murine hematopoietic stem cell subsets are decreased by preincubation with hematopoietic growth factors. Blood 85:2598, 1995

8. Vos O, Buurman WA, Ploemacher RE: Mobilization of haemopoietic stem cells (CFU) into the peripheral blood of the mouse: Effects of endotoxin and other compounds. Cell Tissue Kinet 5:467, 1972 
9. Verstegen MM, van Hennik PB, Terpstra W, van den Bos C, Wielenga JJ, van Rooijen N, Ploemacher RE, Wagemaker G, Wognum AW: Transplantation of human umbilical cord blood cells in macrophagedepleted SCID mice: Evidence for accessory cell involvement in expansion of immature CD34+CD38-cells. Blood 91:1966, 1998

10. Lahiri SK, Putten LMv: Distribution and multiplication of colony forming units from bone marrow and spleen after injection in irradiated mice. Cell Tissue Kinet 2:21, 1969

11. Lord BI: The relationship between spleen colony production and spleen cellularity. Cell Tissue Kinet 4:211, 1971

12. Metcalf D, Moore MAS: General description of blood cells and haemopoietic organs, in Neuberger A, Tatum E (eds): Haemopoietic Cells, vol 1 (ed 1). Amsterdam, The Netherlands, North-Holland, 1971, p 1

13. Hardy CL, Megason GC: Specificity of hematopoietic stem cell homing. Hematol Oncol 14:17, 1996

14. Kataoka M, Tavassoli M: Identification of lectin-like substances recognizing galactosyl residues of glycoconjugates on the plasma membrane of marrow sinus endothelium. Blood 65:1163, 1985

15. Aizawa S, Tavassoli M: Marrow uptake of galactosyl-containing neoglycoproteins: Implications in stem cell homing. Exp Hematol 16:811, 1988

16. Aizawa S, Tavassoli M: Molecular basis of the recognition of intravenously transplanted hemopoietic cells by bone marrow. Proc Natl Acad Sci USA 85:3180, 1988

17. Williams DA, Rios M, Stephens C, Patel VP: Fibronectin and VLA-4 in haematopoietic stem cell-microenvironment interactions. Nature 352:438, 1991

18. Teixido J, Hemler ME, Greenberger JS, Anklesaria P: Role of beta 1 and beta 2 integrins in the adhesion of human CD34hi stem cells to bone marrow stroma. J Clin Invest 90:358, 1992

19. Vermeulen M, Le Pesteur F, Gagnerault MC, Mary JY, Sainteny F, Lepault F: Role of adhesion molecules in the homing and mobilization of murine hematopoietic stem and progenitor cells. Blood 92:894, 1998

20. Papayannopoulou T, Craddock C, Nakamoto B, Priestley GV, Wolf NS: The VLA4/VCAM-1 adhesion pathway defines contrasting mechanisms of lodgement of transplanted murine hemopoietic progeni- tors between bone marrow and spleen. Proc Natl Acad Sci USA 92:9647, 1995

21. Ploemacher RE, Brons NH, van Soest PL: Colony formation by bone marrow cells after incubation with neuraminidase. III. Cell surface repair, homing and growth characteristics of colony forming cells in vivo. Exp Hematol 9:168, 1981

22. Spertini O, Kansas GS, Reimann KA, Mackay CR, Tedder TF: Function and evolutionary conservation of distinct epitopes on the leukocyte adhesion molecule-1 (TQ-1, Leu-8) that regulate leukocyte migration. J Immunol 147:942, 1991

23. Walcheck B, White M, Kurk S, Kishimoto TK, Jutila MA: Characterization of the bovine peripheral lymph node homing receptor: A lectin cell adhesion molecule (LECAM). Eur J Immunol 22:469, 1992

24. Sgroi D, Stamenkovic I: The B-cell adhesion molecule CD22 is cross-species reactive and recognizes distinct sialoglycoproteins on different functional T-cell sub-populations. Scand J Immunol 39:433, 1994

25. Saeland S, Duvert V, Caux C, Pandrau D, Favre C, Valle A, Durand I, Charbord P, de Vries J, Banchereau J: Distribution of surface-membrane molecules on bone marrow and cord blood CD34+ hematopoietic cells. Exp Hematol 20:24, 1992

26. Watanabe T, Dave B, Heimann DG, Lethaby E, Kessinger A, Talmadge JE: GM-CSF-mobilized peripheral blood CD34+ cells differ from steady-state bone marrow CD34+ cells in adhesion molecule expression. Bone Marrow Transplant 19:1175, 1997

27. Timeus F, Crescenzio N, Basso G, Ramenghi U, Saracco P, Gabutti V: Cell adhesion molecule expression in cord blood CD34+ cells. Stem Cells 16:120, 1998

28. Aizawa S, Tavassoli M: Detection of membrane lectins on the surface of hemopoietic progenitor cells and their changing pattern during differentiation. Exp Hematol 16:325, 1988

29. van Hennik PB, Verstegen MM, Bierhuizen MF, Limon A, Wognum AW, Cancelas JA, Barquinero J, Ploemacher RE, Wagemaker G: Highly efficient transduction of the green fluorescent protein gene in human umbilical cord blood stem cells capable of cobblestone formation in long-term cultures and multilineage engraftment of immunodeficient mice. Blood 92:4013, 1998 